\title{
Article
}

\section{Hot and cold knees: exploring differences in patella skin temperature in patients with patellofemoral pain}

Janssen, Jessica, Selfe, James, Gichuru, Phillip, Richards, James, Yosmaoglu, Hayri Baran, Sonmezer, Emel, Erande, Renuka, Resteghini, Peter and Dey, Paola

Available at http://clok.uclan.ac.uk/33148/

Janssen, Jessica ORCID: 0000-0002-5961-2736, Selfe, James, Gichuru, Phillip, Richards, James ORCID: 0000-0002-4004-3115, Yosmaoglu, Hayri Baran, Sonmezer, Emel, Erande, Renuka, Resteghini, Peter and Dey, Paola (2020) Hot and cold knees: exploring differences in patella skin temperature in patients with patellofemoral pain. Physiotherapy, 108 . pp. 55-62. ISSN 0031-9406

It is advisable to refer to the publisher's version if you intend to cite from the work. http://dx.doi.org/10.1016/j.physio.2020.04.007

For more information about UCLan's research in this area go to

http://www.uclan.ac.uk/researchgroups/ and search for <name of research Group>.

For information about Research generally at UCLan please go to http://www.uclan.ac.uk/research/

All outputs in CLoK are protected by Intellectual Property Rights law, including Copyright law. Copyright, IPR and Moral Rights for the works on this site are retained by the individual authors and/or other copyright owners. Terms and conditions for use of this material are defined in the policies page. 
1 Hot and cold knees: exploring differences in patella skin temperature in patients with

2 patellofemoral pain.

3 Jessie Janssen ${ }^{1,2}$, James Selfe ${ }^{3}$, Phillip Gichuru ${ }^{4}$, Jim Richards ${ }^{1}$, Hayri Baran Yosmaoğlu 5 , Emel

4 Sönmezer ${ }^{5}$, Renuka Erande $^{6}$, Peter Resteghini ${ }^{6}$, Paola Dey $^{4}$

1. Allied Health Research unit, University of Central Lancashire, UK

2. Institute of Therapeutic Sciences, IMC University of Applied Sciences Krems, Austria

3. Department of Health Professions, Manchester Metropolitan University, UK

4. Faculty of Health, Social Care and Medicine, Edge Hill University, Ormskirk, UK

5. Department of Physiotherapy and Rehabilitation, Baskent University, Turkey

6. Physiotherapy Department, Homerton University Hospital NHS Foundation Trust, UK

12 Corresponding author:

13 Dr Jessie Janssen

14 Department of Health Sciences

15 Institute of Therapeutic Sciences

16 IMC University of Applied Sciences Krems

17 Piaristengasse 1

183500 Krems, Austria

19 Telephone: +432732 - 802 - 165

20 E-Mail: jessie.janssen@fh-krems.ac.at 


\section{Abstract}

2 Objectives: to investigate the distribution of patella skin temperature (Tsk) measurements and to explore the presence of temperature subgroups in patellofemoral pain (PFP)

4 patients.

5 Design: cross-sectional observational study design

6 Participants: One dataset of 58 healthy participants and 232 PFP patients from three

7 different datasets.

8 Main outcome measures: Patella skin temperature, measured by physiotherapists using a

9 low cost hand held digital thermometer. The distribution of patella skin temperature was assessed and compared across datasets. To objectively determine the clinically meaningful number of subgroups, we used the average silhouette method. Finite mixture models were then used to examine the presence of PFP temperature subgroups. Receiver operating characteristic curves were used to estimate optimal patella Tsk thresholds for allocation of participants into the identified subgroups.

Results: In contrast to healthy participants, the patella skin temperature had an obvious bimodal distribution with wide dispersion present across all three PFP datasets. The fitted finite mixture model suggested three temperature subgroups (cold, normal and hot) that had been recommended by the average silhouette method with discrimination cut-off thresholds for subgroup membership based on receiver operating curve analysis of Cold $=<30.0^{\circ} \mathrm{C}$; Normal $30.0-35.2^{\circ} \mathrm{C}$; Hot $\geq 35.2^{\circ} \mathrm{C}$.

Conclusion: A low cost hand held digital thermometer appears to be a useful clinical tool to identify three PFP temperature subgroups. Further research is recommended to deepen 
1 understanding of these clinical findings and to explore the implications to different 2 treatments. 


\section{Contribution of the Paper:}

2 - A low cost hand held digital thermometer can be used to identify patellofemoral

3 pain temperature subgroups

4

- These three skin temperature subgroups appear to be: Cold $\leq 30^{\circ} \mathrm{C}$; Normal 30 -

5 $35.5^{\circ} \mathrm{C} ;$ Hot $\geq 35.5^{\circ} \mathrm{C}$.

6

7 Key Words:

8 - Patellofemoral pain

9 - Skin temperature

10 - Subgroups

11 - ROC analysis

12

13

14

15

16

17

18

19

20 


\section{Introduction}

People with patellofemoral pain (PFP) present with anterior retropatellar and/or peripatellar pain during squatting, running and going up and down the stairs [1]. The prevalence of PFP has been estimated to be between $1.5 \%$ and $7.3 \%$ of all patients seeking medical care $[2,3]$. The current best evidence-based treatment approach for PFP is multimodal (a combination of treatments), which can include knee and hip muscle strengthening and lengthening, patellar taping, patellar mobilizations, and foot orthoses [4]. However, there is limited evidence to support the longer-term outcomes of this treatment approach [5-8]. In view of the limited success of the multimodal approach, support for the concept of clinically subgrouping PFP patients and delivering targeted treatment has grown and has been supported as a priority area of investigation by consecutive International PFP Research Retreats [9-12]. As a result, some clinically based PFP subgrouping studies are now beginning to emerge [13-16], but to date these have focussed on biomechanical imaging and or psychological factors.

However, there is evidence that physiological factors, such as vascularisation of the knee, could play an important role in PFP. A small number of investigators, using sophisticated laboratory methods, such as photoplethysmography, have suggested that PFP in some patients may be induced by ischemia [17-19]. In these patients, knee skin temperature (Tsk) is thought to be reduced due to poor circulation. However, a more recent study by van der Heijden et al in 2016 [20] using Quantitative Dynamic Contrast-Enhanced Magnetic Resonance Imaging (DCE-MRI) found no difference in perfusion parameters of the patellar bone between PFP patients and healthy control subjects. In other conditions, the same dilemma exists. Coughlan et al [21], and more recently McConnell [22], reported decreased knee Tsk as a feature of PFP complex regional pain syndrome (CRPS). In contrast, O'Brien 
1 [23] reported that a warm knee, detected through thermal imaging and confirmed by

2 arteriography, may be associated with cases of arterial occlusion at the popliteal fossa.

3 Therefore, there is some laboratory and clinical evidence suggesting that a variety of

4 circulatory issues may result in pain and altered temperature profiles around the patella and

5 the knee.

6 From anatomical and biomechanical perspectives, the vessels of both the arterial supply and

7 venous drainage of the patella are located in the soft tissues on the anterior aspect of the

8 knee, situated anterior to the axis of knee flexion. Therefore, during activities of daily living

9 requiring knee flexion, such as, sitting and stair climbing, these vessels are stressed and subjected to vascular torsion or bending, which could lead to intermittent occlusion and ischaemia resulting in hypoxia. As outlined above, intermittent ischaemia may manifest clinically as cold and painful knees, and a number of clinical studies have reported that cold knees can be associated with PFP. For example, Sandow and Goodfellow [24] reported that $16.7 \%(n=9)$ of a sample of adolescent females had pain that was aggravated by cold weather and Price et al [25] described how out of 46 chronic, traumatic anterior knee pain patients $61 \%$ had pain that was aggravated by cold weather.

Selfe et al $[26,27]$ investigated cold knees in PFP patients using patient questioning, therapist palpation and thermal imaging. Collectively the results suggested there may be a small subgroup of PFP patients who complain of painful cold knees, and have measurable Tsk reductions over the patella, and importantly have worse outcomes in response to conventional multimodal physiotherapy treatment $[26,27]$. A limitation of these observations for clinical practice is the high cost of infra-red thermal imaging cameras which inhibits there use in routine clinical environments. However, a low-cost method of 
assessment of Tsk at the knee using a hand-held digital thermometer has recently been

2 validated against a thermal imaging camera [28].

3 Over the last 5 years, the authors have undertaken four studies in which patella Tsk was

4 measured in the same standardised way using the same model of digital thermometer, the

5 FORA IR19 infrared thermometer (ForaCare, Suisse, AG, Neugasse 55, 9000 St. Gallen,

6 Switzerland) previously known as an IR 21 B digital thermometer (RDSM nv, Hasselt,

7 Belgium). Healthy volunteers were tested in one study and patients with PFP attending

8 physiotherapy clinics were tested in a further three studies. In this paper, we report on the

9 distribution of Tsk across the four studies and explore the possibility of the existence of PFP temperature subgroups.

\section{Methods}

Design

A cross-sectional observational study design was used. No formal power calculation was undertaken given the exploratory nature of the study.

\section{Description of datasets}

The healthy volunteer study (normative dataset) recruited 71 participants without back or lower limb problems and without a history of knee surgery. This healthy volunteer study was a validation study of the digital thermometer used in all three PFP studies against thermal imaging measurements, and its methods are reported in detail in Erande et al [28]. To facilitate the comparison between the healthy subjects and PFP patients, only data from 58 knees of the 29 participants who were aged between 18 and 40 years were included. 
1 Of the three studies undertaken in newly diagnosed PFP patients, two were undertaken in

2 the United Kingdom (UK): London [28] and the UK wide TIPPs (Targeted Interventions for

3 Patellofemoral Pain study) [15], and one in Turkey [29]. All three studies used the same participant eligibility criteria used in previous studies [30,31]. Patients were included if the

5 duration of their pain was at least 3 months and they self-reported anterior or retropatellar

6 pain during at least two of the following activities: prolonged sitting, ascending or

7 descending stairs, squatting, running, kneeling and hopping/jumping. Two of the following

8 factors were also required from a clinical examination: pain during resisted isometric

9 quadriceps contraction, pain on palpation of the posterior facets of the patella, pain during squatting. Patients with specific pathologies, such as, ligamentous instability or patella subluxation were excluded. Participants were included irrespective of whether they had unilateral or bilateral patella pain.

In the TIPPs study, data from 127 patients, aged 18 to 40 years attending one of four National Health Service physiotherapy clinics, were analysed to explore subgroups in the PFP population. The study methods are reported in Selfe et al [15] and these participants have also been included in the current study. The second UK study (London) was part of a postgraduate research project to compare digital temperature measurements with measures of tissue oxygenation, and recruited 24 PFP patients aged 18 to 40 years who attended an NHS physiotherapy clinic in London between December 2014 and September 2015.

The Turkish study was undertaken in Baskent University physiotherapy clinic, Ankara. In total 85 participants were recruited between May 2015 and May 2017, of whom 81 had 
patella Tsk measurements. Methods are reported in Yosmaoğlu et al [29]. In addition, to

2

temperature, the PFP datasets included information on age, sex, bilateral pain, ambient room temperature, patella Tsk and tibialis anterior muscle Tsk. Two datasets also included information on body mass index and time since clinical onset of pain.

5

\section{Temperature measurements}

Where possible, all four studies conformed to the recently published 15-point TISEM consensus statement on the measurement of human Tsk [32]. Participants were asked to change into shorts 15 minutes before the start of data collection, to avoid the Tsk being affected by clothing. Other items on the TISEM checklist such as advice to participants on the effect of exercise, smoking, alcohol, lotions or effects in clinical environment on Tsk were not standardised, however, clinicians and participants were briefed prior to commencement of the study about these factors and were asked to consider and/or avoid these where possible. In all three studies of PFP patients, Tsk over the muscle belly of the tibialis anterior (tibialis anterior muscle) was measured contemporaneously with patella Tsk using the same digital thermometer; ambient room temperature was also measured. In all studies, participants attended a single visit for the assessment. In the case of the PFP patients, this was prior to the commencement of physiotherapy treatment, an experienced physiotherapist undertook the temperature measurements; standardised training and a reference manual was provided across all sites.

The FORA IR19 infrared thermometers (ForaCare, Suisse, AG, Neugasse 55, 9000 St. Gallen, Switzerland) used across all sites are compliant with the accuracy requirement specification ASTM E1965.98 and the EC directive 93/42/EEC. The displayed temperature range was $23^{\circ} \mathrm{C}$ 
1 to $44^{\circ} \mathrm{C}$, and the manufacturers stated a display resolution of $0.1^{\circ} \mathrm{C}$ and an accuracy of

$2 \pm 0.3^{\circ} \mathrm{C}$ for the range between $35.0^{\circ} \mathrm{C}$ and $41.0^{\circ} \mathrm{C}$. The thermometer was originally designed

3 for measuring foot temperature to aid the management of diabetic foot problems, the

4 temperature sensing element does not touch the skin, however, the rim of the

5 thermometer is in contact with the skin.

6

7 In all studies, once they had changed into shorts, participants were given 15 minutes to

8 acclimatise to the room temperature, allowing their lower limb Tsk to stabilise [33], no

9 physical interventions or physiotherapy techniques were conducted or performed prior to this. For the temperature measurements, subjects were relaxed in supine lying with the legs

11 extended in the neutral anatomical position. An average of three readings was taken at the centre of the patella in both knees in all participants (Figure 1a). In addition, in the PFP studies, an average of 3 readings was taken over the muscle belly of the tibialis anterior $10 \mathrm{~cm}$ distal to the tibial tubercle and $2 \mathrm{~cm}$ lateral to the anterior border of the tibia (Figure 1b). During these measurements, the thermometer was held so the sensor was perpendicular to the skin. Ambient room temperature was also recorded contemporaneously. 


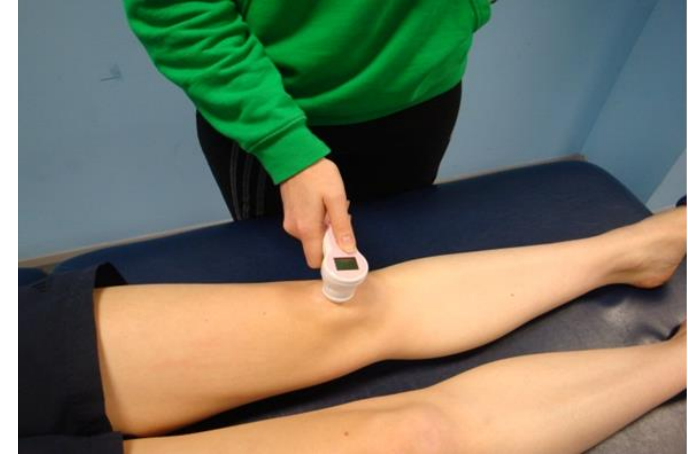

Figure 1a. Patella temperature

measurement

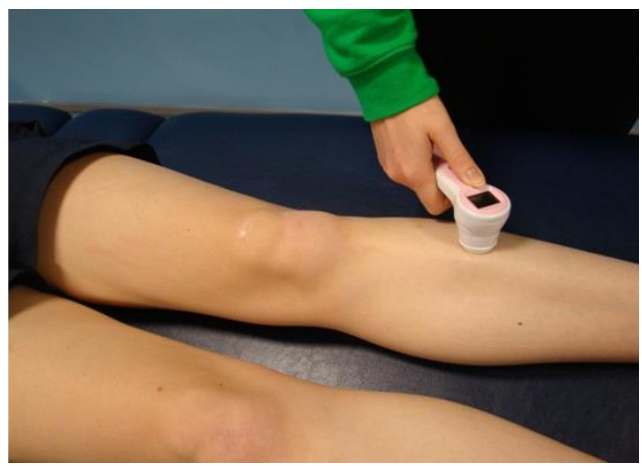

Figure 1b. Tibialis anterior muscle Tsk measurement

Ethics:

3 The studies conformed to the World Medical Declaration of Helsinki, research on human participants. All relevant institutional, health department and governmental approvals were

5 in place prior to data collection at each site. Information on approvals can be found in

6 Erande et al [28] for the thermometer validation study and Selfe et al [15] for the TIPPs

7 study. Ethical approval for the London study was obtained from the Regional ethics committee (REC 14/10/1388) and local university ethics committee (STEMH 274). For the

9 Turkish study, ethical approval was obtained from the local Turkish University clinical research discussion committee (KA 15/18). Written Informed consent was obtained for all studies.

Statistical Analysis

Participant characteristics for age, tibialis anterior muscle Tsk, sex, body mass index, time since clinical onset of pain and presence of bilateral PFP for each study were summarised by means (standard deviation) or percentages as appropriate. Patellar Tsk (for all datasets) and tibialis anterior muscle Tsk distribution characteristics (for the three PFP datasets) were 
explored graphically (using histogram plots) and formally to assess the extent of adherence to the normal distribution. To assess the strength of the relationship between the Tsk temperatures and ambient room temperature, scatter plots of the pairs of variables complemented by either Pearson or Spearman correlation were calculated, as appropriate, depending on the degree of observed skewness of the PFP distributions in the datasets.

The presence of subgroups was explored by merging the PFP datasets and using a finite 'mixture' models to explore the presence of clinically meaningful temperature subgroups. The average silhouette method as described by Kaufman and Rousseeuw [34] was used to identify the ideal number of subgroups. The mixture models are probabilistic models that capture the presence of subpopulations within an overall population, often without succinct knowledge of the actual sub-population group to which each individual belongs [35]. Formally, a 'mixture' model corresponds to the mixture distribution that represents the probability distribution of observations in the overall population and are used to make statistical inferences about the properties of sub-populations given only observations on the pooled population, without sub-population identity information.

The fitted three component finite mixture model was used to assign each participant to a particular subgroup based on the maximum posterior probability value. A comparison of means of ambient room temperature was performed using an ANOVA analysis across the identified subgroups. If an overall statistically significant $(p<0.05)$ difference was detected, multiple comparisons between subgroups were performed using Tukey's B (Wholly Significant Difference) test while also carrying out the Games-Howell test to check if the observed subgroup variances differed substantially. Receiver operating characteristic (ROC) 
curves were then used to identify optimal cut-off thresholds between observed subgroups, using maximum sensitivity and specificity to discriminate between subgroups.

\section{Results}

The participant characteristics are shown in Table 1. Mean ages were similar but there were more males in the normative dataset compared with the three PFP datasets. The sex distribution was similar across the three PFP studies, but the frequency of bilateral symptoms differed.

Table 1: Participant demographics and clinical and temperature characteristics

\begin{tabular}{|c|c|c|c|c|}
\hline & \multicolumn{4}{|c|}{ Datasets } \\
\hline & Normative & TIPPS & Turkish & London \\
\hline Number of participants ( $\mathrm{N}$ ) & 29 & 127 & 81 & 24 \\
\hline Number of knees & 58 & 127 & 81 & 24 \\
\hline \multicolumn{5}{|l|}{ Patient characteristics } \\
\hline Mean age in years (SD) & $28.7(6.4)$ & $26.5(5.7)$ & $26.1(6.6)$ & $30.7(5.2)$ \\
\hline Female $\mathrm{n}(\%)$ & $11(38 \%)$ & $84(66 \%)$ & $63(74 \%)$ & $16(67 \%)$ \\
\hline Bilateral n (\%) & Not relevant & $67(53 \%)$ & $29(34 \%)$ & $17(71 \%)$ \\
\hline Mean BMI kg/m²(SD) & Not available & $25.4(5.8)$ & 22.5 (3.9) & $\begin{array}{r}\text { Not } \\
\text { available }\end{array}$ \\
\hline Mean time from onset in months (SD) & Not relevant & $46.0(55.8)$ & $25.2(33.2)$ & $\begin{array}{r}\text { Not } \\
\text { available }\end{array}$ \\
\hline \multicolumn{5}{|l|}{ Temperature measures } \\
\hline $\begin{array}{l}\text { Mean tibialis anterior muscle skin } \\
\text { temperature }{ }^{\circ} \mathrm{C}(\mathrm{SD})\end{array}$ & Not available & $35.8(0.7)$ & $36.0(0.7)$ & $36.2(0.2)$ \\
\hline $\begin{array}{l}\text { Mean ambient room temperature }{ }^{\circ} \mathrm{C} \\
\text { (SD) }\end{array}$ & Not available & $24.0(1.5)$ & $24.8(2.3)$ & $24.5(0.8)$ \\
\hline
\end{tabular}

SD-Standard Deviation, ${ }^{0} \mathrm{C}$-degrees centigrade, TIPPS-Targeted Interventions for Patellofemoral Pain study, BMI-Body Mass Index

Figure 2 shows the distribution of patella Tsk in the normative dataset and each of the PFP datasets. The type of distribution differed between the normative dataset which appeared unimodal with a relatively narrow dispersion (mean $30.2^{\circ} \mathrm{C}$ (SD 1.5 ), median $30.0^{\circ} \mathrm{C}$ (IQR 29.1 -30.9); Figure 2a) and the three PFP datasets, which all appeared to have a bimodal 
1 distribution with a wider dispersion of values compared with the normative dataset (TIPPs

2 median $30.7^{\circ} \mathrm{C}$ (IQR $27.6-35.6$ ), Turkish median $34.4^{\circ} \mathrm{C}$ (IQR 31.7 to 35.8 ), London median

$3 \quad 31.9^{\circ} \mathrm{C}$ (IQR 27.9 to 35.4$)$ ). The Turkish dataset had fewer values at the lower end of the

4 temperature scale. In contrast, to this bimodal distribution for patella Tsk, the tibialis

5 anterior muscle Tsk in the three PFP datasets were unimodal with a narrow range of values

6 (Figure 3). 

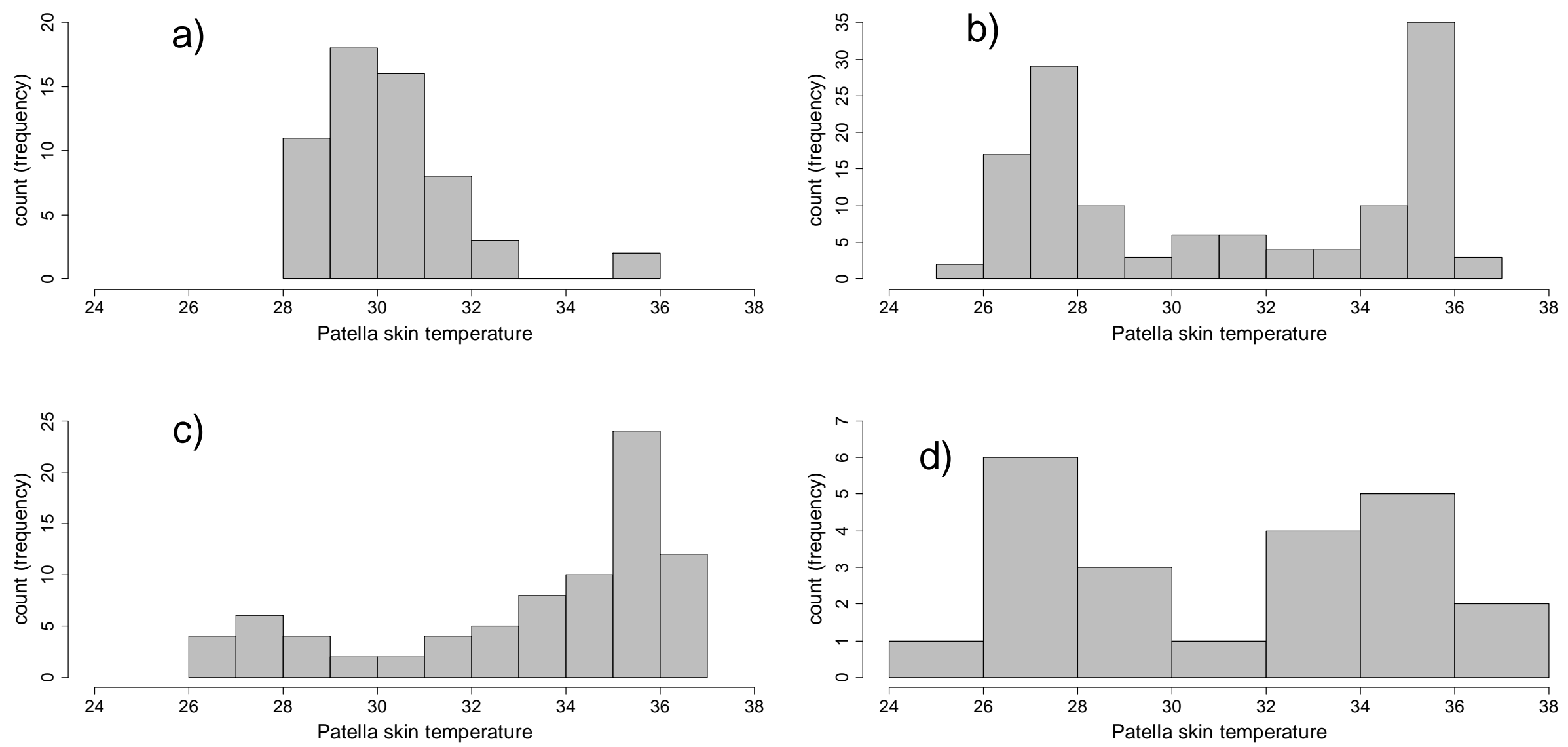

Figure 2: Histogram plots for patella skin temperature in the PFP datasets: a) Normative b) TIPPs c) Turkish d) London. 

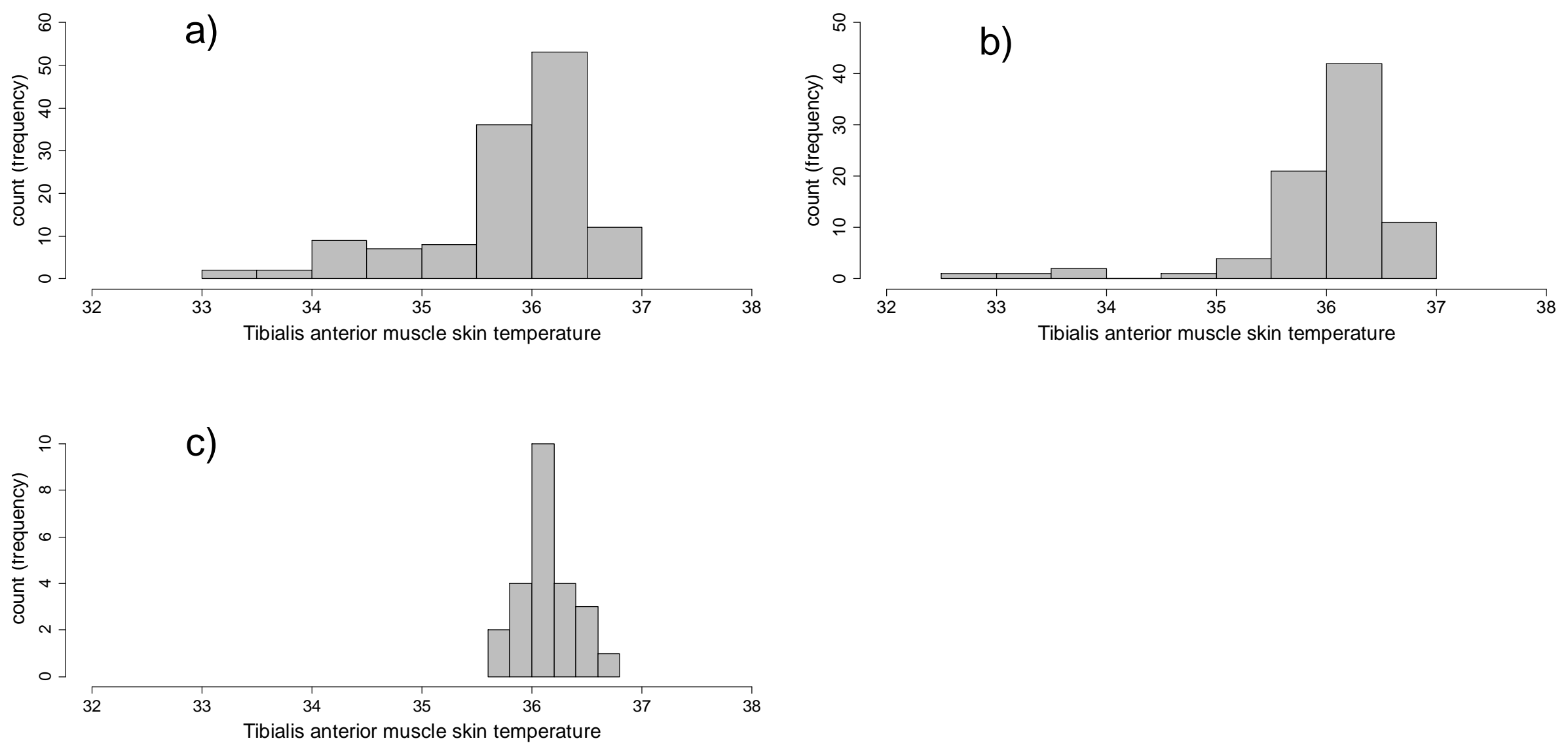

Figure 3: Histogram plots for tibialis anterior muscle skin temperature in the PFP datasets: a) TIPPs b) Turkish c) London. 
Mean tibialis anterior muscle Tsk and mean ambient room temperature were similar across the three datasets (Table 1). There was no correlation between tibialis anterior muscle Tsk and ambient room temperature (Pearson $\mathrm{R}=-0.01, \mathrm{p}=0.89$ ) but there was a weak positive statistically significant correlation between patella Tsk and ambient room temperature (Spearman $\mathrm{R}=0.32, \mathrm{p}<0.001$ ).

The bimodal distribution observed in the PFP participants suggested three potential temperature subgroups: these were designated as a cold subgroup, a normal subgroup and a hot subgroup. To explore this further the three PFP datasets were merged. Three subgroups were suggested as optimal by the elbow plot (supplementary Figure 1). Finite mixture models were applied, which identified subgroup memberships for each participant. The mean (SD) patella Tsk for the cold subgroup was $27.5^{\circ} \mathrm{C}(0.8)$, for the normal subgroup was $33.2^{\circ} \mathrm{C}(1.8)$ and for the hot subgroup was $35.8^{\circ} \mathrm{C}(0.8)$ as obtained from the fitted finite mixture model. The ROC curve analyses revealed a cut-point value of $30.0^{\circ} \mathrm{C}$ with $100 \%$ sensitivity and 99\% specificity to discriminate between "cold" and "not cold" knees and a cut-off value of $35.2^{\circ} \mathrm{C}$ with $100 \%$ sensitivity and $99 \%$ specificity to discriminate between "hot" and "not hot" knees respectively (Figure 4). 


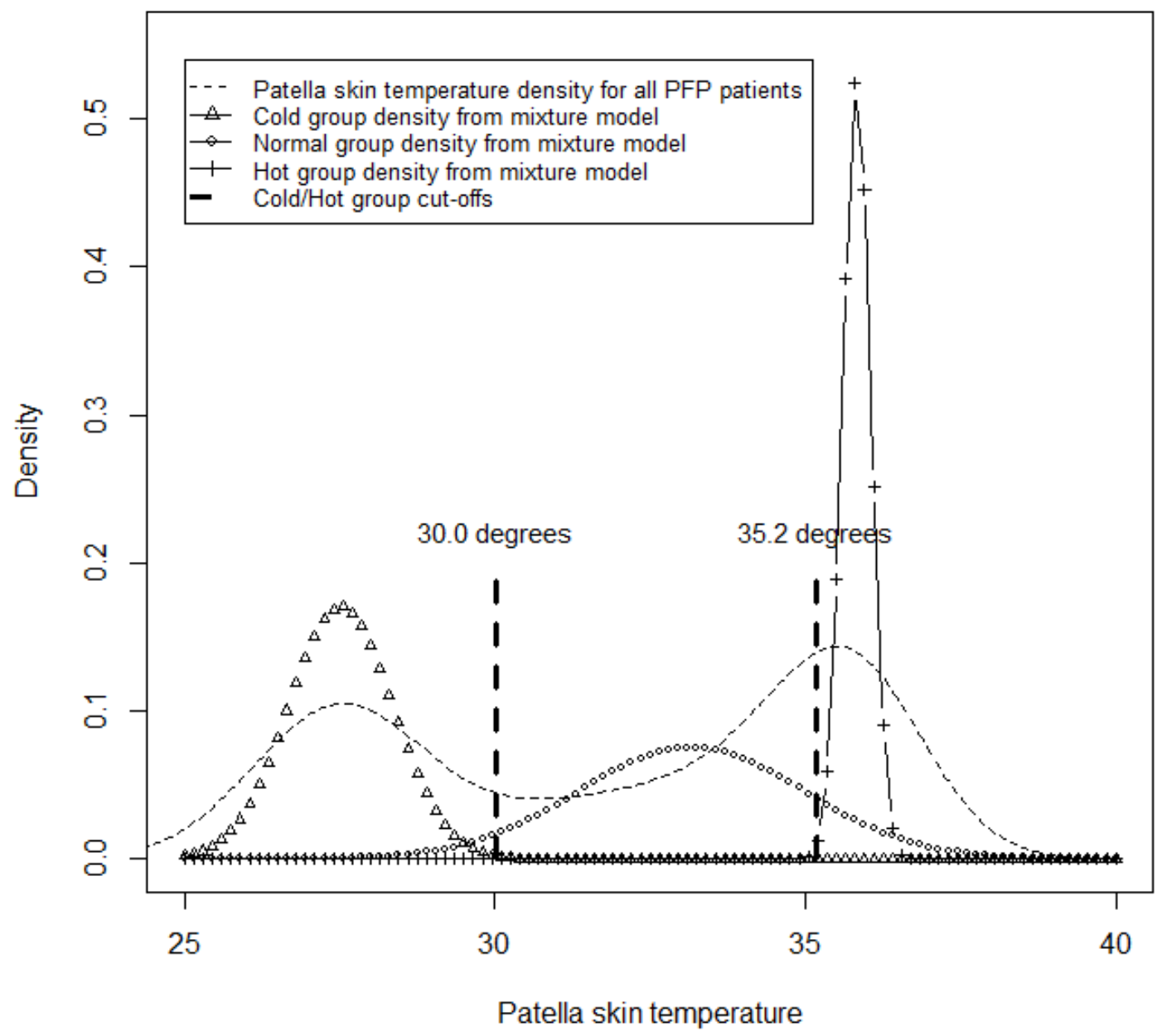

Figure 4: Patella skin temperature bimodal distribution for all the 232 PFP patients from the three PFP studies

As ambient room temperature was weakly correlated with patella Tsk, we further explored the distribution of ambient room temperature across the subgroups. The cold, normal and hot subgroups had mean ambient room temperatures of $23.6^{\circ} \mathrm{C}(1.5), 24.8^{\circ} \mathrm{C}(1.7)$ and $24.7^{\circ} \mathrm{C}(2.1)$ respectively. There was a statistically significant difference in ambient room temperature across the three subgroups $(F=12.83, p<0.001)$ but from a pairwise 
comparison, this was limited to significant differences between the cold subgroup and the two other subgroups (vs hot subgroup: difference $=-1.2^{\circ} \mathrm{C}, \mathrm{p}<0.001$; vs normal subgroup: difference $\left.=-1.3^{\circ} \mathrm{C}, \mathrm{p}<0.001\right)$. There was no difference between the normal and the hot subgroup (difference $\left.=0.1^{\circ} \mathrm{C}, \mathrm{p}=0.995\right)$.

\section{Discussion}

This study explored the patella Tsk in healthy subjects and patients with PFP. These results suggest that the Tsk over the centre of the patella in PFP patients has a bimodal distribution which is distinct compared to the unimodal distribution displayed by those without PFP. Further analysis suggested that there are 3 temperature subgroups (cold, normal and hot) and subsequent ROC analysis suggested optimal cut point thresholds for subgroup membership, which in routine practice would be set at $\leq 30^{\circ} \mathrm{C}$ for the cold subgroup and $\geq 35.5^{\circ} \mathrm{C}$ for the hot subgroup allowing for the accuracy of the digital thermometer. The mean temperature of the healthy participants and tibialis anterior muscle in PFP participants lie within the range of values of the patella Tsk of the normal subgroup. The classification of three temperature subgroups map well to the published data on the knee. Ammer [36] in his review of the temperature of the human knee based on contact temperature measurements reported that the mean temperature of the anterior knee in 876 healthy subjects was $30.5^{\circ} \mathrm{C} \pm 1.1$. Ammer also reported a higher temperature of $32.5^{\circ} \mathrm{C}$ \pm 0.9 in 512 inflammatory rheumatoid arthritis patients. Therefore, patients in the hot subgroup could have an inflammation undetected in clinical practice, as was discovered by Zampeli et al. [37] using microwave radiometry. In contrast, patients in the lowest temperature subgroup could have intermittent ischaemia or poor vascularisation [17-19] 
The cold subgroup is consistent with our previous study [27] in PFP patients using a noncontact thermal imaging camera which found a lower mean patella baseline Tsk of $28.4^{\circ} \mathrm{C}$ (SD 1.3) in the 'cold group' compared to $29.7^{\circ} \mathrm{C}$ (SD 1.6) in the 'not cold group'. Other authors have also observed an abnormal reaction to environmental cold in some patients with anterior knee pain $[24,25]$. In our study, the ambient room temperature was lower among the cold subgroup than the other two subgroups, suggesting that the participants may be responding to the cooler temperatures. The cooler temperatures did not have an impact on the tibialis anterior skin temperature, suggesting that this was a local patellar response. This response to external temperatures may in part explain why the Turkish participants had a higher median patella Tsk with fewer patients within the lower range of temperature values compared to the two United Kingdom datasets, albeit with similar tibialis anterior mean temperatures. There might also be other environmental or ethnic differences which could contribute to these differences between countries, but there is a dearth of research on such impacts on the incidence, aetiology or outcome of PFP.

There were sex differences in distribution between the healthy and the PFP participants, but it is well recorded that females are more likely to present with PFP $[38,39]$. There were also differences in presence of bilateral PFP between the PFP datasets, but despite this the presence of bimodal temperature distribution was observed in all three PFP datasets. The impact of patient and clinical characteristics on subgroup membership and on differences between Tsk distribution in healthy and patellofemoral pain groups warrants further investigation as this may provide further insights into the natural history and aetiology of these subgroups.

Strengths and limitations 
This study is one of the largest studies in patellofemoral pain, and has been achieved through the collaboration of research teams utilising the same methods and equipment. All the physiotherapy clinics in the three patient studies provided a full range of musculoskeletal services and served local general populations, therefore all these participants are likely to represent PFP patients within the community rather than special populations such as elite athletes or the military [38]. This increases the generalisability of our findings to routine clinical practice. Further generalisability of the study to clinical practice was achieved through the use of a low cost hand-held digital thermometer to identify the subgroups.

These pragmatic, clinically based studies were not able to be undertaken under standardised conditions for room temperature, contrary to some of the recommendations presented in the TISEM checklist [32]. However, as described above, this ambient room temperature variation may have helped reveal the presence of a cold subgroup, a group of PFP patients who respond to cooler temperatures. The hot subgroup did not respond to variations in ambient room temperature. Information on the other items on the TISEM checklist, such as the effect of exercise, smoking, alcohol, lotions or effects in clinical environment on Tsk, was provided, however future studies could more closely monitor these factors.

These were cross-sectional studies and they cannot provide information on the possible reasons for subgroup membership. It may be that the pathogenesis differs between the subgroups, for example that the cold subgroup have intermittent ischaemia and the hot subgroup have inflammation, or it could be that this represents a continuum of disease progression. Nor can it provide information on whether the different subgroups differ in 
their long-term outcome. Further research would require longitudinal designs and collection of a broader range of patient and clinical characteristics, mechanistic information and outcome data.

\section{Conclusion}

Three subgroups of PFP patients based on Tsk over the patella have been proposed, and thresholds for subgroup membership based on ROC curve analysis suggested, these are: Cold $\leq 30^{\circ} \mathrm{C}$; Normal $30-35.5^{\circ} \mathrm{C}$; Hot $\geq 35.5^{\circ} \mathrm{C}$. These subgroups map well to the limited previous literature in this field $[27,36]$. This study demonstrates high ecological validity as a low-cost hand-held digital thermometer appears to be a useful clinical tool to identify these groups. However, further research, more closely aligned to the TISEM guidelines, should be undertaken to confirm the study findings with more information about patient demographic and clinical characteristics and a well-matched healthy comparator group. Following this further research should investigate the aetiology of these subgroups and whether identifying these subgroups based on Tsk helps the targeting of interventions and the improvement of outcomes for PFP patients. 


\section{Ethical approval}

Information on approvals can be found in Erande et al [28] for the thermometer validation study and Selfe et al [15] for the TIPPs study. Ethical approval for the London study was obtained from the Regional ethics committee (REC 14/10/1388) and local university ethics committee (STEMH 274). For the Turkish study, ethical approval was obtained from the local Turkish University clinical research discussion committee (KA 15/18).

\section{Sources of Funding}

The TIPPs study was funded by Arthritis Research UK (grant 19950). The study at Baskent University, Turkey was mainly funded by The Scientific and Technological Research Council of Turkey (TUBITAK) and an Erasmus plus grant.

\section{Conflict of interest}

There are no conflicts of interest.

\section{References}

[1] Collins NJ, Vicenzino B, van der Heijden RA, van Middelkoop M. Pain during prolonged sitting is a common problem in persons with patellofemoral pain. J Orthop Sports Phys Ther. 2016;46:658-663. https://doi. org/10.2519/jospt.2016.6470

[2] Smith BE, Selfe J, Thacker D, Hendrick P, Bateman M, Moffatt F, Rathleff M, Smith T, Logan P (2018) Incidence and prevalence of patellofemoral pain: A systematic review and meta-analysis. PLoS ONE 2018;13(1):11

[3] Glaviano NR, Kew M, Hart JM, Saliba S. Demographic and epidemiological trends in patellofemoral pain. Int J Sports Phys Ther. 2015;10:281-290. 
[4] Willy RW, Hoglund LT, Barton CJ, Bolgla LA, Scalzitti DA, Logerstedt DS, Lynch AD, SnyderMackler L, McDonough CM. Patellofemoral Pain. J Orthop Sports Phys Ther. 2019 Sep;49(9):CPG1-CPG95. doi: 10.2519/jospt.2019.0302.

[5] Crossley K, Bennell K, Green S, Cowan S, McConnell J. Physical therapy for patellofemoral pain: a randomized, double-blinded, placebo-controlled trial. Am J Sports Med. 2002 NovDec;30(6):857-65.

[6] Whittingham M, Palmer S, Macmillan F. Effects of taping on pain and function in patellofemoral pain syndrome: a randomized controlled trial. J Orthop Sports Phys Ther. 2004;34(9):504-10.

[7] Dixit S, DiFiori JP, Burton M, Mines B. Management of patellofemoral pain syndrome. Am Fam Physician. 2007 15;75(2):194-202.

[8] Blond L, Hansen L. Patellofemoral Pain Syndrome in Athletes. Acta Orthop Belg. 1998;64:393-400.

[9] Davis I, Powers C. Patellofemoral Pain Syndrome: Proximal, Distal, and Local Factors. An International Retreat. JOSPT. 2010;40(3):A1-48

[10] Powers CM, Bolgla LA, Callaghan MJ, Collins N, Sheehan FT. Patellofemoral pain: proximal, distal, and local factors, $2^{\text {nd }}$ International Research Retreat. J Orthop Sports Phys Ther. 2012 Jun;42(6):A1-54.

[11] Witvrouw E, Callaghan MJ, Stefanik JJ, Noehren B, Bazett-Jones DM, Willson JD, EarlBoehm JE, Davis IS, Powers CM, McConnell J, Crossley KM. Patellofemoral pain: consensus statement from the 3rd International Patellofemoral Pain Research Retreat held in Vancouver, September 2013. Br J Sports Med. 2014 Mar;48(6):411-4 
[12] Crossley KM, Stefanik JJ, Selfe J, Collins NJ, Davis IS, Powers CM, McConnell J, Vicenzino B, Bazett-Jones DM, Esculier JF, Morrissey D, Callaghan MJ. 2016 Patellofemoral pain consensus statement from the 4th International Patellofemoral Pain Research Retreat, Manchester. Part 1: Terminology, definitions, clinical examination, natural history, patellofemoral osteoarthritis and patient-reported outcome measures. Br J Sports Med. 2016 Jul;50(14):839-43.

[13] Selhorst M, Rice W, Degenhart T, Jackowski M, Tatman M. Evaluation of a treatment algorithm for patients with patellofemoral pain syndrome: a pilot study. Int J Sports Phys Ther. 2015;10:178-188

[14] Keays S, Mason M, Newcombe P. Individualised physiotherapy in the treatment of patellofemoral pain. Physiother Pract Res. 2015;20:22-36.

[15] Selfe J, Janssen J, Callaghan M, Witvrouw E, Sutton C, Richards J, Stokes M, Martin D, Dixon J, Hogarth R, Baltzopoulos V, Ritchie E, Arden N, Dey P. Are there three main subgroups within the patellofemoral pain population? A detailed characterisation study of 127 patients to help develop targeted intervention (TIPPs). Br J Sports Med. 2016 Jul;50(14):873-80. doi:10.1136/bjsports-2015-094792

[16] Drew B, Conaghan P, Smith T, Selfe J, Redmond A, Grainger A, Hensor E, Dube B. Development of data-driven diagnostic subgroups for people with patellofemoral pain using modifiable clinical, biomechanical and imaging features. JOSPT. 201949 (7):536-547

[17] Arnoldi CC. The patellar pain syndrome. Acta Orthop Scand 1991;62(Suppl244):1-29.

[18] Sanchis-Alfonso V, Rosello-Sastre E. Anterior knee pain in the young patient-what causes the pain?"Neural model”. Acta Orthop Scand. 2003;74(6):697-703. 
[19] Naslund J, Walden M, Lindberg LG. Decreased pulsatile bloodflow in the patella in patellofemoral pain syndrome. Am J Sports Med. 2007;35:1668-73.

[20] van der Heijden RA, Poot DHJ, Ekinci M, Kotek G, van Veldhoven PL, Klein S, Verhaar JAN, Krestin GP, Bierma-Zeinstra SMA, van Middelkoop M, Oei EHG. Blood perfusion of patellar bone measured by dynamic contrast-enhanced MRI in patients with patellofemoral pain: A case-control study. J Magn Reson Imaging. 2018 Nov;48(5):1344-1350.

[21] Coughlan RJ, Hazleman BL, Page Thomas DP, Sattelle L, Crisp AJ, Jenner JR, Dandy DJ. Algodystrophy: A Common Unrecognized Cause Of Chronic Knee Pain. Br J Rheumatol. $1987 ; 26: 270-274$.

[22] McConnell J Chapter 2: Patients emotional states and expectations. In Patellofemoral Pain: An evidence-based clinical guide. Eds Selfe J, Janssen J, Callaghan M. Nova Science. New York, 2017

[23] O'Brien DP, Walsh TN, Given HF. The warm knee sign-an evaluation. Eur J Vasc Surg. 1990 Oct;4(5):531-4.

[24] Sandow MJ, Goodfellow JW. The natural history of anterior knee pain in adolescents. J Bone Jt Surg (Br). 1985;67:36-38

[25] Price AJ, Jones J, Allum R. Chronic traumatic anterior knee pain. Injury. 2000;31;373-378

[26] Selfe, J Harper L, Pedersen I, Breen-Turner J, Waring J, Stevens D. Cold legs: A potential indicator of negative outcome in the rehabilitation of patients with patellofemoral pain syndrome? Knee. 2003;10(2):139-143

[27] Selfe J, Sutton C, Hardaker N, Greenhalgh S, Karki A, Dey P. Anterior knee pain and cold knees: a possible association in women. Knee. 2010;17:319-323. 
[28] Erande R, Dey M, Richards J, Selfe J. An investigation of the relationship between thermal imaging and digital thermometer testing at the knee. Physiother Pract Res. 2016 $37(1): 41-47$

[29] Yosmaoğlu HB, Selfe J, Sonmezer E, Sahin IE, Duygu SÇ, Acar Ozkoslu M, Richards J, Janssen J. Targeted Treatment Protocol in Patellofemoral Pain: Does Treatment Designed According to Subgroups Improve Clinical Outcomes in Patients Unresponsive to Multimodal Treatment? Sports Health. 2019 Nov; 21:1941738119883272.

[30] Syme G, Rowe P, Martin D, et al (2009) Disability in patients with chronic patellofemoral pain syndrome: A randomised controlled trial of VMO selective training versus general quadriceps training. Man Ther. 2009;14: 252-263

[31] Cook C, Hegedus E, Hawkins R, Scovell F, Wyland D. Diagnostic accuracy and association to disability of clinical test findings associated with patellofemoral pain syndrome. Physiother Can. 2010 Winter;62(1):17-24.

[32] Moreira DG, Costello JT, Brito CJ, Adamczyk JG, Ammer K, Bach AJE, Costa CMA, Eglin C, Fernandes AA, Fernández-Cuevas I, Ferreira JJA, Formenti D, Fournet D, Havenith G, Howell K, Jung A, Kenny GP, Kolosovas-Machuca ES, Maley MJ, Merla A, Pascoe DD, Priego Quesada JI, Schwartz RG, Seixas ARD, Selfe J, Vainer BG, Sillero-Quintana M. Thermographic imaging in sports and exercise medicine: A Delphi study and consensus statement on the measurement of human skin temperature. J Therm Biol. 2017 Oct;69:155-162. doi: 10.1016/j.jtherbio.2017.07.006.

[33] Mayr H. Thermographic evaluation after knee surgery. In Ammer K, Ring E FJ (Eds). The Thermal Image in Medicine and Biology . Uhlen-Verlag, Wien. 1995 
[34] Kaufman L, Rousseeuw P. Finding Groups in Data: An Introduction to Cluster Analysis. John Wiley \& Sons, Inc., Hoboken, New Jersey. 1990

[35] Benaglia T, Hunter DR, Young DS. mixtools: An R Package for Analyzing Finite Mixture Models. Journal of Statistical Software. 2009 October;32(6):1-29.

[36] Ammer K. Temperature of the human knee - a review. Thermology international 2012; 22(4):137-151.

[37] Zampeli E, Raftakis I, Michelongona A, Nikolaou C, Elezoglou A, Toutouzas K, Siores E, Sfikakis PP. Detection of subclinical synovial inflammation by microwave radiometry. PLoS One. 2013;May 31;8(5):e64606. doi: 10.1371/journal.pone.0064606.

[38] Boling M, Padua D, Marshall S, Guskiewicz K, Pyne S, Beutler A. Gender differences in the incidence and prevalence of patellofemoral pain syndrome. Scand J Med Sci Sports. 2010 Oct;20(5):725-30.

[39] Smith BE, Selfe J, Thacker D, Hendrick P, Bateman M, Moffatt F, Rathleff MS, Smith TO, Logan, P. Incidence and prevalence of patellofemoral pain: A systematic review and metaanalysis. PloS one. 2018;13(1), e0190892. doi:10.1371/journal.pone.0190892 


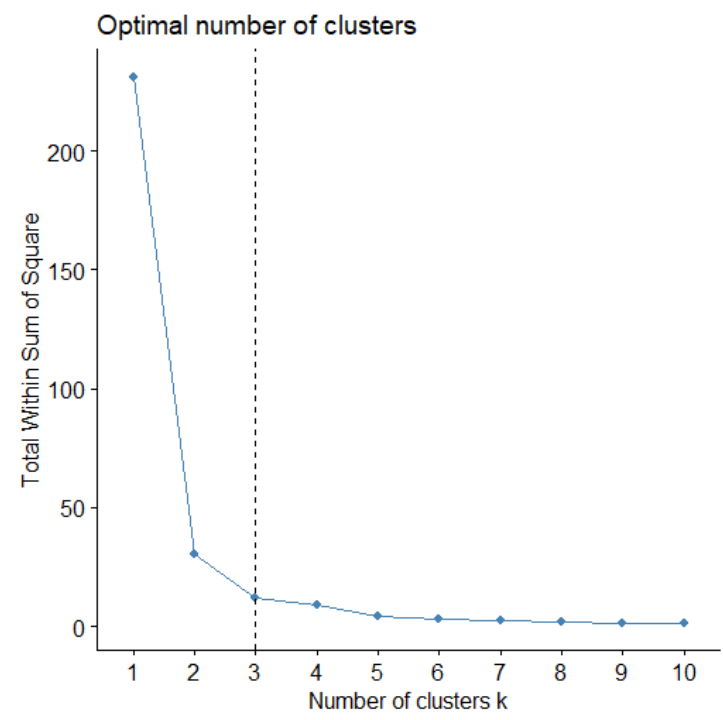

Supplementary Figure 1: Elbow plots to determine the optimal number of clusters in the patella skin temperature for all PFP studies combined. 